\title{
Multipole seismoelectric logging while drilling (LWD) for acoustic velocity measurements
}

\author{
Zhenya Zhu', Jun Wang ${ }^{1,2}$, and M.N. Toks ö $z^{1}$ \\ ${ }^{1}$ Earth Resources Laboratory \\ Dept. of Earth, Atmospheric, and Planetary Sciences \\ Massachusetts Institute of Technology, Cambridge, MA 02139 \\ ${ }^{2}$ Department of Astronautics and Mechanics \\ Harbin Institute of Technology, Harbin, China, 150001.
}

\begin{abstract}
In seismoelectric well logging, an acoustic wave propagates along a borehole and induces electrical signals along the borehole wall. The apparent velocities of these seismoelectric signals are equal to the formation velocities. Laboratory scale-model multipole acoustic and seismoelectric LWD tools are built to conduct measurements in a borehole drilled into a sandstone formation. The tools include either an acoustic receiver array of an electrode receiver array along with four acoustic sources to allow the generation of monopole, dipole, and quadrupole modes. Results show that the standard acoustic measurement of formation velocities are impacted by strong tool wave contamination in most situations. However, because the propagating tool waves do not induce any electrical signals, the seismoelectric measurements can provide a more robust velocity measurement. The multipole seismoelectric logging-whiledrilling (LWD) could be used as a new logging method to measure the acoustic velocities of the borehole formations.
\end{abstract}

\section{Introduction}

Multipole (monopole, dipole, and quadrupole) acoustic well logging while drilling (LWD) was developed in directional or horizontal drilling (Rao and Vandiver, 1999; Tang et al., 2002; Wang 
and Tang, 2003; Chi et al., 2005). The source and receiver transducers are tightly mounted on the drill collar; the coupling is stiffer than a wireline tool (Zhu et al., 2008b). As a result, very strong tool waves are generated by a monopole, dipole, or quadrupole source; these waves propagate along the collar with the different velocities and are called tool waves. The tool wave arrivals can interfere with the formation arrivals making accurate $\mathrm{P}$ and $\mathrm{S}$ wave velocity estimation difficult. Significant effort is spent trying to remove or attenuate these tool waves with varying degrees of success. We present an alternative method of measuring the formation arrivals using electrode receiver arrays to measure seismoelectric waves that propagate at the formation $\mathrm{P}$ and $\mathrm{S}$ wave velocities. These seismoeletric signals are not impacted by tool waves and therefore provide a more robust way to make acoustic LWD measurements.

When a porous medium is saturated with an electrolyte solution, an electric double layer (EDL) is formed on the interface between the solid and the fluid (Morgan et al., 1989; Pride, 1994; Jardani et al., 2010). Some ions are absorbed on the solid surface and other ions remain movable in the fluid. When a seismic wave propagates in the fluid-saturated porous medium, the seismic wave generates relative movement between the solid and the fluid. The movement of the ions in the fluid forms an electric current and a seismoelectric field. When the fluid flows through a sample at a certain pressure, the fluid flow induces a current and we can measure the resultant voltage.

Experiments in borehole models (Zhu and Toksöz, 1998; Zhu et al., 1999) show that two different kinds of electric fields are induced by a Stoneley wave in fluid-saturated fractured or layered boreholes. Experiments measure two kinds of seismoelectric fields predicted by theoretical studies (Fenoglio et al., 1995; Pride and Haartsen, 1996; Garambois and Dietrich, 2001). The first seismoelectric field is the electric and magnetic field induced by the propagation of a seismic wave in a homogeneous porous medium; the second seismoelectric field is the EM wave generated at an interface or through a heterogeneous medium (Zhu et al., 2008a). We refer to the first field as the stationary or localized seismoelectric field and the second one as the radiating EM wave or seismoelectromagnetic wave (Zhu and Toksöz, 2003, 2005).

In this paper we conduct seismoelectric measurements in a water tank and a layered borehole model to show the basic properties of the seismoelectric conversion in a rock sample and a borehole model. We build a scaled multipole acoustic LWD tool to perform measurements in a water tank and in a sandstone borehole model to show the strong tool waves that are generated. We also build a multipole seismoelectric LWD tool to measure the seismoelectric field in the borehole model and observe that the tool wave has no effect on the received electric signals.

\section{Seismoelectric measurements in a water tank}

We conduct seismoelectric measurements with different samples in a water tank. A power hydrophone (Celesco LC-34) in a water tank is excited with a single sine burst $(70 \mathrm{kHz}$ in center frequency) (Figure 1). A sample plate (using different materials) is placed in the water tank with a distance of $21.5 \mathrm{~cm}$ from the source. An electrode and a receiver hydrophone (B \& K 8103) are 
placed in front of the sample. We record the acoustic waveform and the electric signals. First we record the electric field of the background when there is no sample in the water tank. We then put Lucite, aluminum, limestone, and Berea sandstone samples into the location shown in Figure 1 , respectively. The acoustic waveform and the electric signals are recorded with the acoustic receiver and the electrode as shown in Figure 2. The seismoelectric signals cannot be induced by the acoustic wave in solid Lucite and metal (aluminum). The arrival times of the electric signals recorded with limestone and sandstone samples, show that the acoustic wave induces the seismoelectric signals in these porous rocks. The amplitude of the electric signal induced in sandstone is higher than that in limestone, because the porosity of the sandstone sample is higher than that of the limestone sample. Because metal is a good conductor, when a metal sample is in the water or is connected with the ground, the entire metal sample keeps the same potential and no seismoelectric potential can be induced.

\section{Seismoelectric measurements in a layered borehole model}

We conduct both acoustic and seismoelectric measurements in a layered borehole model (Figure $3)$. The porosity and permeability of slate are very low and those of epoxy-glued sand are very high. An acoustic source is fixed in the slate section and is excited with an electric pulse to generate an acoustic field in the borehole. An acoustic hydrophone (B \& K 8103) or an electrode moves along the water-filled borehole and across the interface between the slate and glued sand to record the acoustic or electric signals (Figure 4). Comparing the arrival times or the slope of the seismoelectric signals in Figure $4 \mathrm{~b}$ with those of the acoustic waves (Stoneley waves) in Figure 4a, we know that the seismoelectric signals are induced by the acoustic waves propagating in the formations and along the borehole. The seismoelectric signals are the localized or stationary seismoelectric field and the apparent velocity is equal to the acoustic velocity in the formation. The amplitudes are sensitive to the formation porosity and permeability. The amplitudes of the seismoelectric signals in the glued-sand section are larger than those in the slate section, even though the acoustic amplitudes in the glued-sand section are lower than those in the slate section. The seismoelectric coupling coefficients in the two sections are different due to their different porosity and permeability. The center frequency of the acoustic waves is higher than that of the seismoelectric signals, because the frequency of the recorded seismoelectric signals is relative to the pore structures of the porous rocks. The amplitudes of the seismoelectric signals, in general, are much lower than those of the acoustic waves due to the seismoelectric voltage coupling coefficients.

\section{Acoustic logging while drilling (LWD) in a scaled borehole}

We build a scaled multipole acoustic LWD tool and conduct measurements with a borehole model in a water tank to investigate the acoustic field in the LWD measurements (Zhu et al., 2008b). 


\section{Scaled multipole acoustic LWD tool}

Figure 5 shows the scaled tool including the source, the connector, and the receiver sections. The source is composed with 4 PZT (Lead zirconate titanate) crystal disks. The source can be worked at different modes of monopole, dipole, and quadrupole (Figure 5) by different connections of the PZT polarizations. At the receiver section, 6 pairs of PZT crystal disks can record the acoustic components and the monopole, dipole, and quadrupole waves can be formed, respectively.

The connector can tightly connect the source and the receiver sections to simulate the acoustic LWD working mode. If the source and the receiver section are fixed in the borehole center without the connector, it more closely simulates the wireline measurement mode.

\section{Tool waves in a water tank}

When the scale tool shown in Figure 5 is located in a water tank $(100 \mathrm{~cm} \times 60 \mathrm{~cm} \times 50 \mathrm{~cm})$ (Figure 6), the acoustic waves cannot be received by the receivers. This is because the acoustic radiation of the source mainly is in the horizontal direction. The acoustic waves reflected by the walls of the water tank can be easily separated in the time domain. Because the source and receivers are tightly connected together, a strong acoustic guided wave propagates along the tool and is received by the receivers - we refer to this as a tool wave. When the source generates different modes, the velocities of the tool waves are different.

Figure 7 shows the received acoustic waveforms and the time-domain semblance plots (Kimball and Marazetta, 1984), when the source operates as a monopole, dipole, and quadrupole source. From the semblances in Figure 7, we see the velocities of the monopole, dipole and quadrupole tool waves are $3100 \mathrm{~m} / \mathrm{s}, 1000 \mathrm{~m} / \mathrm{s}$, and $3100 \mathrm{~m} / \mathrm{s}$, respectively. The center frequencies of the tool waves are slightly different. The frequency of the dipole tool wave is the lowest one and the frequency of the quadrupole tool is the highest one among the tool waves.

\section{Acoustic wireline and LWD measurements in a borehole model}

We next conduct acoustic well logging in an homogeneous sandstone borehole model to investigate the effects of the tool wave on the LWD measurements. The sandstone P-wave velocity is $4700 \mathrm{~m} / \mathrm{s}$ and the S-wave velocity is $2680 \mathrm{~m} / \mathrm{s}$. The diameter of the borehole is 1.65 $\mathrm{cm}$. The borehole model is fully saturated with water. We measure the multipole acoustic fields in the borehole with and without the connector to simulate the LWD and wireline well logging respectively. The waveforms are recorded and the velocities are analyzed with a time-domain semblance method.

(1) Measurements without the tool connector

The tool shown in Figure 5 is separated into two parts: the source and the receiver sections are fixed in the center of the borehole with the same separation $(7.1 \mathrm{~cm})$ as shown in Figure 5. The 
main acoustic energy enters into the formation and propagates along the borehole wall. Because there is no the connector, no acoustic energy can directly propagate along the tool. This measurement is similar to wireline well logging.

Figure 9 shows the acoustic waveforms generated by monopole (a), dipole (b), and quadrupole (c) sources. The velocities can be determined with the time-domain semblance. When the source works as a monopole source the P-wave and S-wave and their velocities are shown in Figure 9a. The Stoneley wave with low frequency is not recorded because the frequency range in this experiment is higher than the Stoneley wave frequency. When the source works as a dipole source, the tool clearly records the flexural wave, whose velocity is very close to the shear wave velocity of the formation. The quadrupole source excites a screw wave and its velocity is equal to the shear velocity.

These results show that the measurements are similar to the wireline well logging, though the 6 pairs of the receivers are fixed on one pipe. In this case, we record the acoustic waves propagating with the $\mathrm{P}$-wave and $\mathrm{S}$-wave velocities of the formation without any influence of tool waves.

\section{(2) Measurements with the tool connector}

In order to simulate acoustic LWD, we include the connector between the source and the receiver sections and put it into the water-filled borehole (Figure 10). In this case, the source energy enters into the borehole formation, but some of the energy also propagates along the tool through the connector. Because the source is directly mounted on the tool, the tool wave is very strong.

Figure 11 shows the waveforms and their time-domain semblances when the source operates as a monopole (a), dipole (b), and quadrupole (c) source, respectively. When the source is a monopole source (Figure 11a), a strong tool wave is excited and it is difficult to separate the formation P- or S- waves. The velocity of the main acoustic energy is a tool wave propagating at a velocity of $3100 \mathrm{~m} / \mathrm{s}$. When the source is a dipole source, the main energy is a dipole tool wave, whose velocity is about $1000 \mathrm{~m} / \mathrm{s}$, which is much lower than the S-wave velocity (2680 $\mathrm{m} / \mathrm{s})$ or water velocity $(1500 \mathrm{~m} / \mathrm{s})$. We could not record the flexural wave propagating in the formation with the $\mathrm{S}$-wave velocity. When the source is a quadrupole source, both the tool wave $(3100 \mathrm{~m} / \mathrm{S})$ and the screw wave with a S-wave velocity are recorded. The tool wave is an influence, but it can be separated from the screw wave.

Because the source and the receiver of the acoustic LWD tool used in an oil field setting are fixed in one collar, they have a very strong coupling between them. The tool wave propagating along the collar will have an effect on the measurements of formation waves. We can apply many methods to eliminate the tool wave propagating along the collar by changing the acoustic or mechanic impedances of the collar, but it is difficult to remove the tool influence completely. The tool wave influence is the most difficult problem in the acoustic well logging while drilling.

Because the seismoelectric signal cannot be induced in metal, we investigate the seismoelectric logging while drilling. 


\section{Multipole seismoelectric well logging while drilling}

We build a scaled multipole seismoelectric LWD tool, based on the acoustic tool shown in Figure 5. The receiver crystals are replaced with electrodes (Figure 12) and we record the electric field induced by the multipole acoustic source in the sandstone borehole model. The apparent velocities are analyzed with semblance.

\section{Scaled multipole seismoelectric LWD tool}

Since the same acoustic source section is used, the source will again operate as a monopole, dipole, or quadrupole mode by combining the polarizations of the source crystals. The source is excited with a square pulse with about 500 volts, which is about 50-time higher than that used in the acoustic measurements. Because the seismoelectric signals are weak, the signals are averaged about 256 times. Good shielding is applied during the measurements to eliminate the influences of outside electromagnetic fields.

Because the source pulse is about $500 \mathrm{~V}$, it is very difficult to shield this signal completely. The influence of the source pulse is recorded in our measurements. The influence of the source pulse is located near the beginning of the waveforms close to time zero in waveform plots. The influence of the source pulse creates a blind area, which is a strong noise for the signal in this area.

\section{Seismoelectric LWD measurements in sandstone borehole model}

The scale seismoelectric LWD tool is positioned in the sandstone borehole model saturated with water of $0.3 \mathrm{mS} / \mathrm{cm}$ in conductivity (Figure 13). Figure 14 shows the recorded electric signals and semblance plots when the acoustic source is a monopole (a), dipole (b), and quadrupole (c), respectively. From the signals, we can see the strong noise induced by the source pulse at the beginning of the waveform plots. (In acoustic measurements the source pulse can be shielded by the receiver transducers.) The amplitudes of the received signals not only depend on the acoustic amplitudes, but also depend on the rock properties near the electrodes. The apparent velocities are the acoustic velocities because the electric signals are induced by the acoustic waves propagating in the formation.

The received electric signals are analyzed with semblance to calculate the apparent velocities (semblance plots in Figure 14). When the source is a monopole source, we record the apparent Swave velocity $(2680 \mathrm{~m} / \mathrm{s})$ and apparent Stoneley wave velocity $(1480 \mathrm{~m} / \mathrm{s})$. The signals propagating with apparent P-wave velocity is not clear in Figure 14a. There are, probably, two reasons: the signals induced by the P-wave arrive early and are in the blind area of the source pulse, and amplitudes of the P-wave are weak in the hard formation (sandstone). Therefore, the seismoelectric signals induced with the P-wave are too weak to be received. In the dipole and quadrupole cases (Figures $14 \mathrm{~b}$ and c), the signals with the apparent S-wave velocity are recorded very clearly.

In the semblance plots of Figure 14, we do not observe any signals propagating with apparent tool wave velocity. This experimental result confirms that the effects of the strong tool waves are 
completely ruled out, because the tool wave propagating along the metal collar cannot induce any seismoelectric signals.

\section{Conclusions}

The principal properties of the seismoelectric conversion are experimentally studied. Acoustic waves can induce the seismoelectric signals in water-filled porous rocks, but cannot induce them in metal. The seismoelectric signals recorded in seismoelectric well logging are stationary or localized electric signals and their apparent velocity is the acoustic velocity of the formation wall. Using scaled acoustic and seismoelectric LWD tools we conduct measurements in a sandstone borehole model. The acoustic tool records strong tool waves propagating along the tool body, which affect the velocity measurements of the formation. Because the tool waves propagating along the metal collar cannot induce any seismoelectric signal, the seismoelectric logging can record the electric signals induced by the acoustic waves propagating in the formation and their apparent velocities are equal to the acoustic velocities of the formation. Because the seismoelectric measurements can completely rule out the effects of tool wave, the seismoelectric well logging while drilling might be a new method to measure the acoustic velocities in the formation. The seismoelectric measurements also record the seismoelectric waveforms, whose amplitudes and phases can be used to investigate additional rock properties, such as porosity and permeability, in the future.

\section{Acknowledgments}

We thank Dr. D. Burns for his valuable suggestion and useful discussion during this study. This work was supported by the Earth Resources Laboratory Founding Member Consortium.

\section{References}

Fenoglio, M. A., M. J. S. Johnston, and J. D. Byerlee, 1995, Magnetic and electric fields associated with changes in high pore pressure in fault zones: Application to the Loma Prieta ULF emissions: Journal of Geophysical Research, 100, 12 951-12 958.

Chi, S., Z. Zhu, R. Rao, and M. N. Toksöz, 2005, Higher order modes in acoustic logging while drilling: 75th SEG Annual International Meeting, Expanded Abstracts, 324-327. 
Garambois, S. and M. Dietrich, 2001, Seismoelectric wave conversions in porous media: Field measurements and transfer function analysis: Geophysics, 66, 5, 1417-1430.

Jardani, A., A. Revil, E. Slob, and W. Söllner, 2010, Stochastic joint inversion of 2D seismic and seismoelectric signals in linear poroelastic materials: A numerical investigation, Geophysics, 75, 1, N19-N31.

Morgan, F. D., E. R. Williams, and T. R. Madden, 1989, Streaming potential properties of Westerly granite with applications: Journal of Geophysical Research, 94, 12449-12461.

Pride, S., 1994, Govering equations for the coupled electromagnetics and acoustics of porous media: Physical Review B 50, 15678-15696.

Pride, S. R., and M. W. Haartsen, 1996, Electroseismic wave properties: Journal of the Acoustical Society of America, 100, 1301-1315.

Rao, V. N. R., and J. K. Vandiver, 1999, Acoustics of fluid-filled boreholes with pipe: Guided propagation and radiation: Journal of theAcoustical Society ofAmerica, 105, 3057-3066.

Tang, X. M., T.Wang, and D. Patterson, 2002, Multipole acoustic logging while-drilling: 72nd Annual International Meeting, SEG, Expanded Abstracts, 364-367.

Wang, T., and X. Tang, 2003, LWD quadrupole shear measurement in anisotropic formation: $73^{\text {rd }}$ Annual International Meeting, SEG, ExpandedAbstracts, 309-312.

Zhu, Z., and Toksöz, M. N., 1998, Seismoelectric measurements in a fractured borehole model: $68^{\text {th }}$ Annual International Meeting, SEG, Expanded Abstracts.

Zhu, Z., M. W. Haartsen, and M. Nafi Toksöz, 1999, Experimental studies of electrokinetic conversions in fluid-saturated borehole models: Geophysics, 64, 5 P1349-1356.

Zhu, Z., and M. N. Toksöz, 2003, Crosshole seismoelectric measurements in borehole models with fractures: Geophysics, 68, 1519-1524.

Zhu, Z., and M. N. Toksöz, 2005, Seismoelectric and seismomagnetic measurements in fractured borehole models: Geophysics, 70, F45-F51.

Zhu, Z, M. N. Toksöz, and D. R. Burns, 2008a, Electroseismic and seismoelectric measurements of rock samples in a water tank: Geophysics, 73, E153-E164.

Zhu, Z, M. N. Toksöz, R. Rao, and D. R. Burns, 2008b, Experimental studies of monopole, dipole, and quadrupole acoustic logging while drilling (LWD) with scaled borehole models: Geophysics.73, 4, E133-E143. 


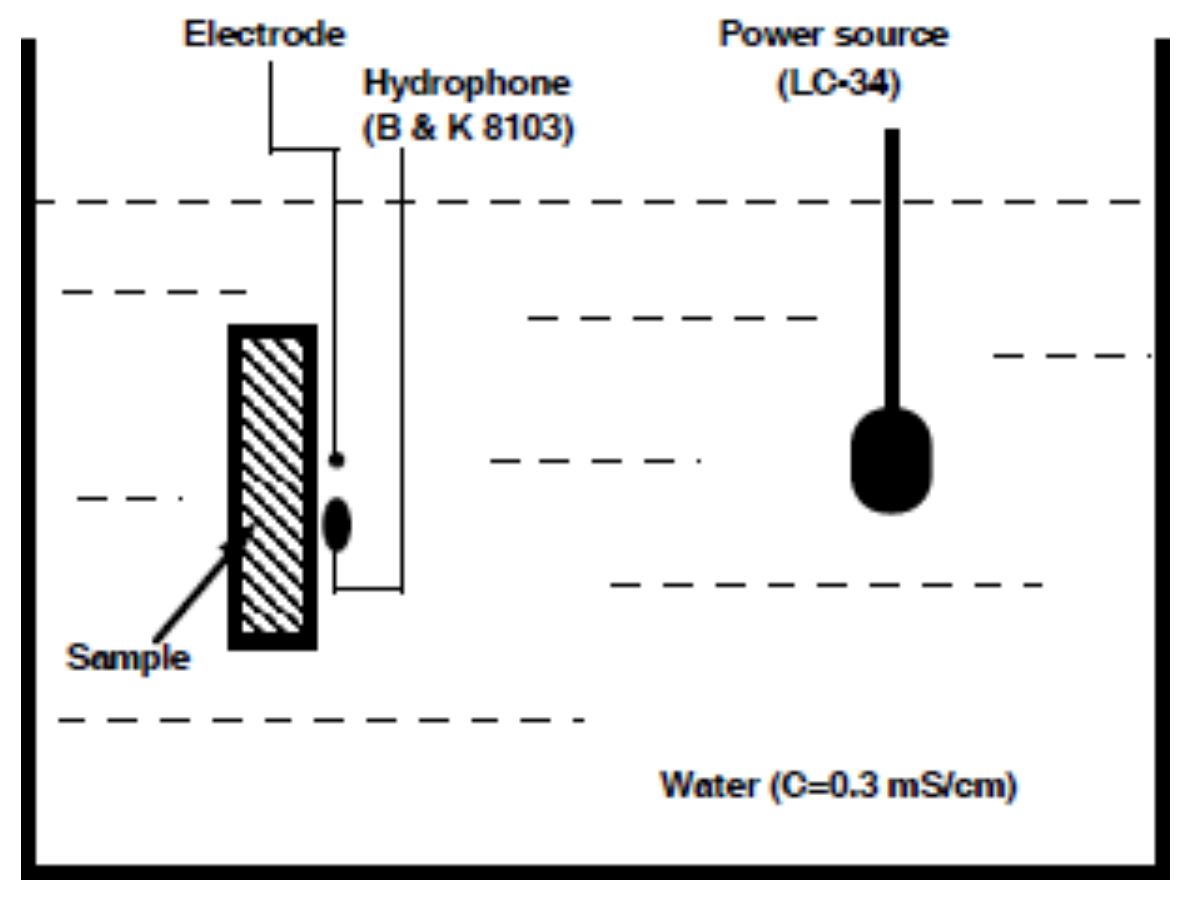

Figure 1: Schematic of seismoelectric measurements in a water tank. A single sine burst with $150 \mathrm{~V}$ and $70 \mathrm{kHz}$ excites the source hydrophone (Celesco LC-34). Measurement electrode records the electric potential close to the sample surface. A receiver hydrophone (B \& K 8103) is placed near the sample to measure the acoustic wave arriving at the sample. 


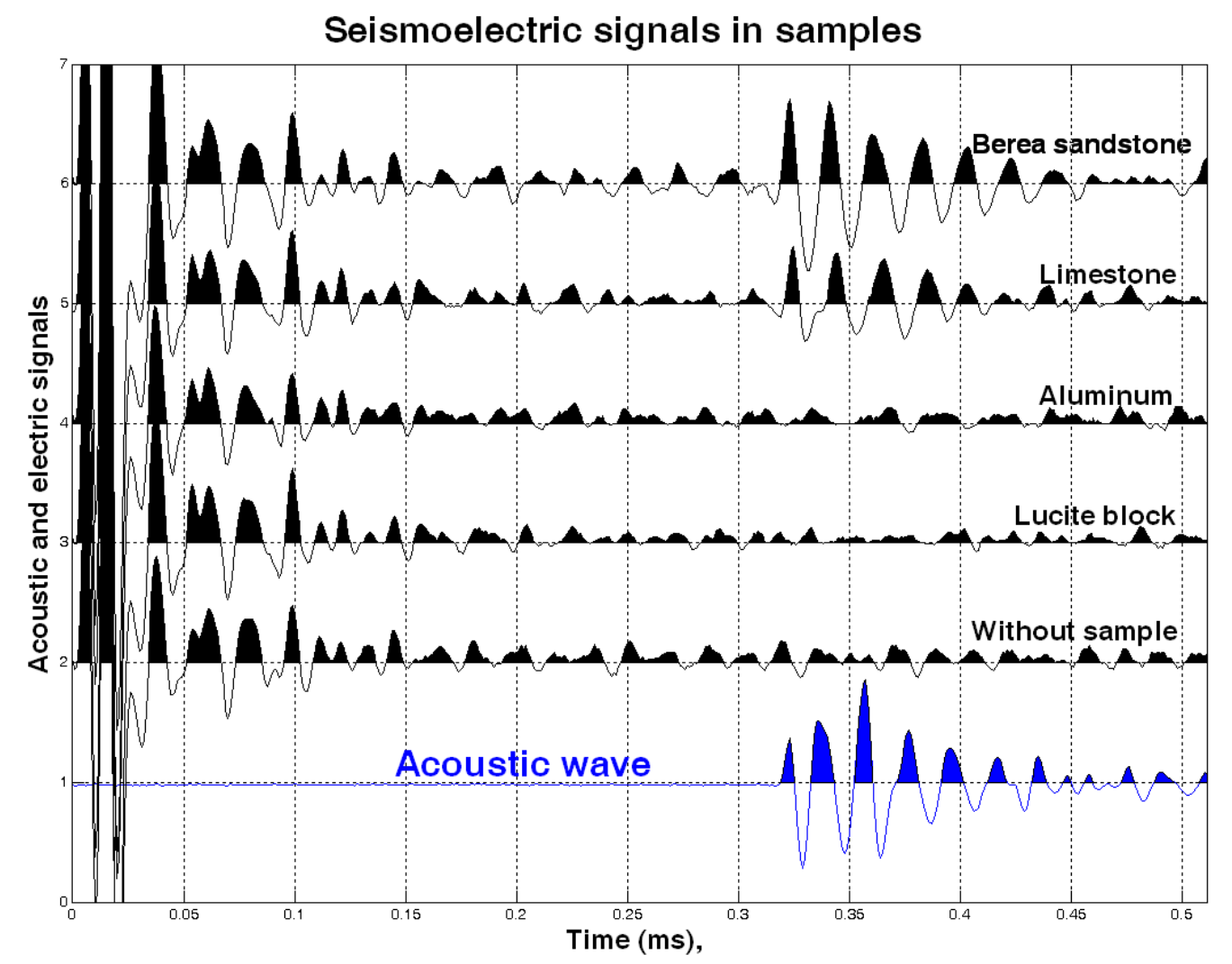

Figure 2: Acoustic waveform (trace 1 with the blue line) and electric signals measured with the different samples. Trace 2 is the electric signal recorded with the electrode when there is no sample. Other traces show electric signals recorded when samples are Lucite (trace 3), aluminum (trace 4), limestone (trace 5), and Berea Sandstone (trace 6), respectively. 


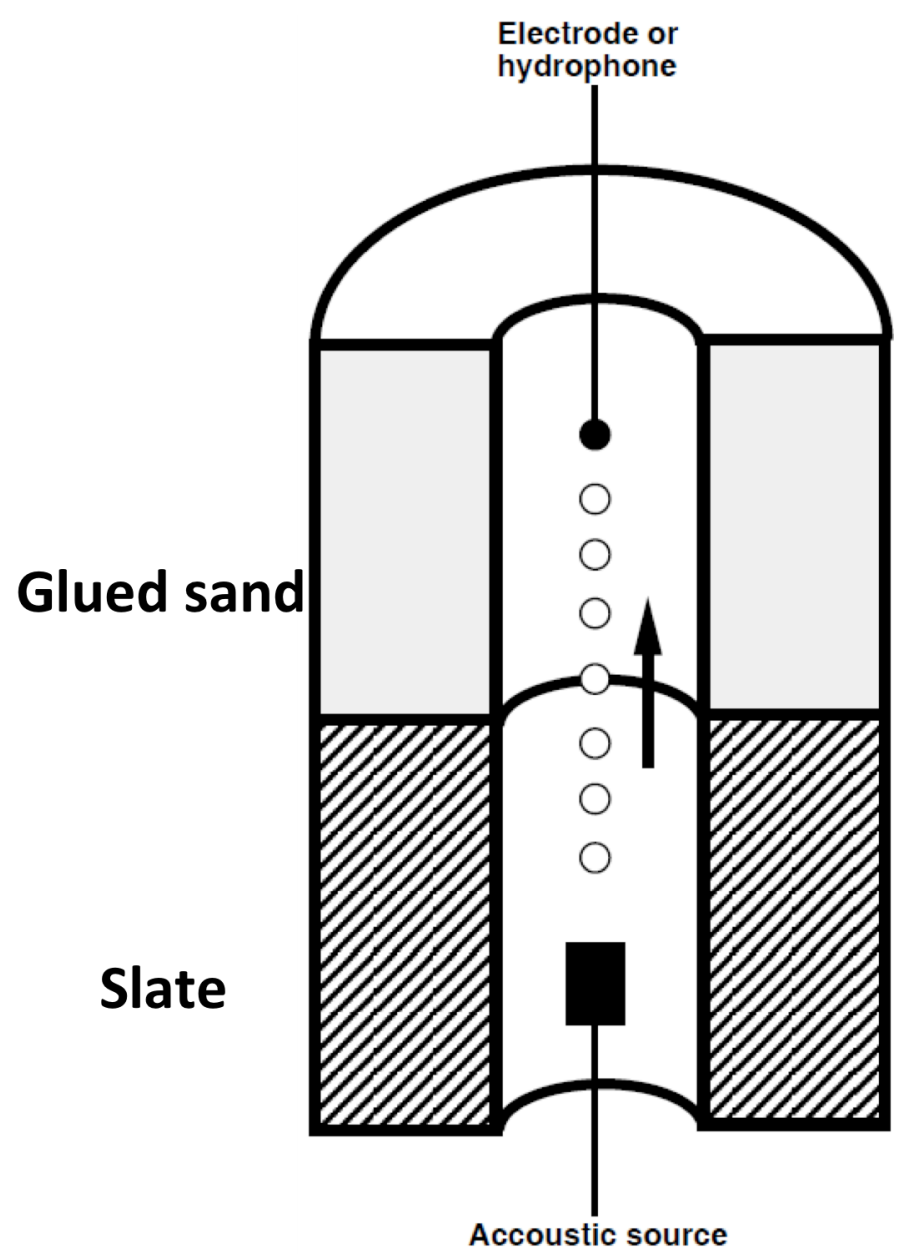

Figure 3: Diagram of the layered borehole model to measure the acoustic field and the seismoelectric field by fixing the acoustic source in the slate section and moving the electrode or acoustic hydrophone along the borehole and across the interface. 
a)

\section{Acoustic waveforms}

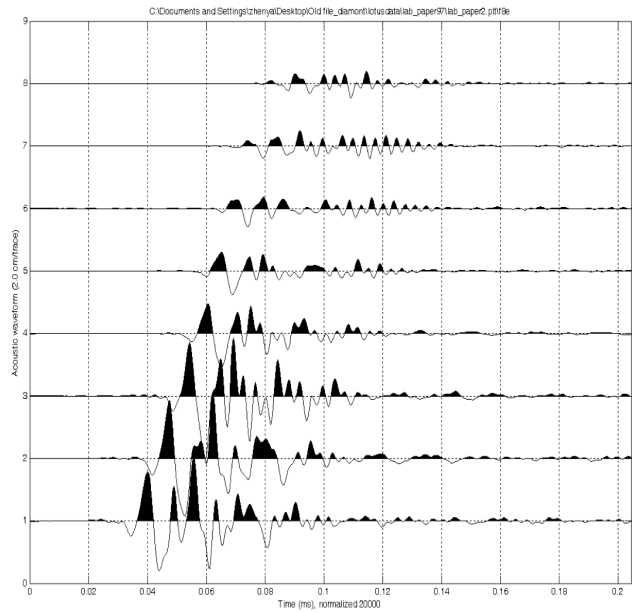

b)

\section{Seismoelectric signals}

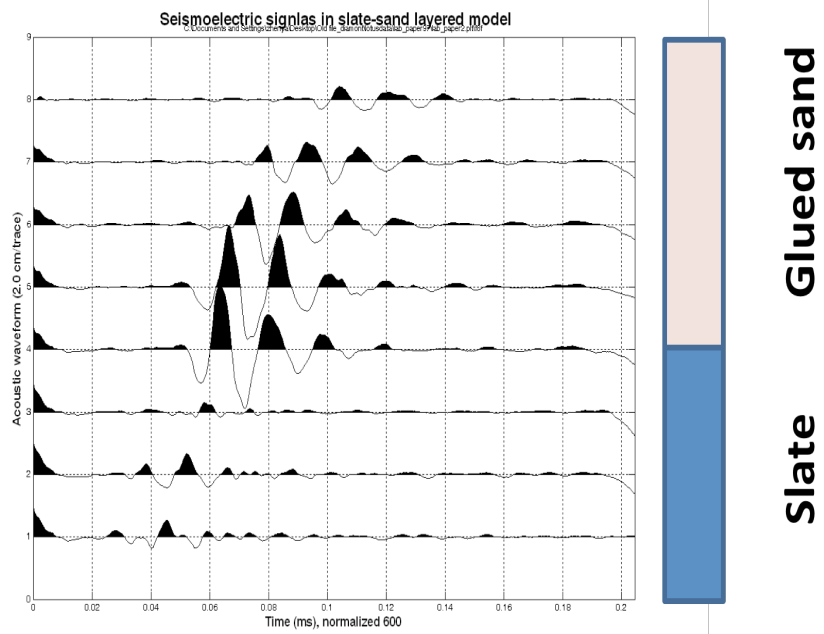

Figure 4: Acoustic waveforms (a) and seismoelectric signals (b) recorded in the borehole model (slate and glued-sand model) when the acoustic source is fixed in the slate section and the acoustic receiver or electrode moves from the slate section to glued-sand section. The fifth trace is located at the interface. 


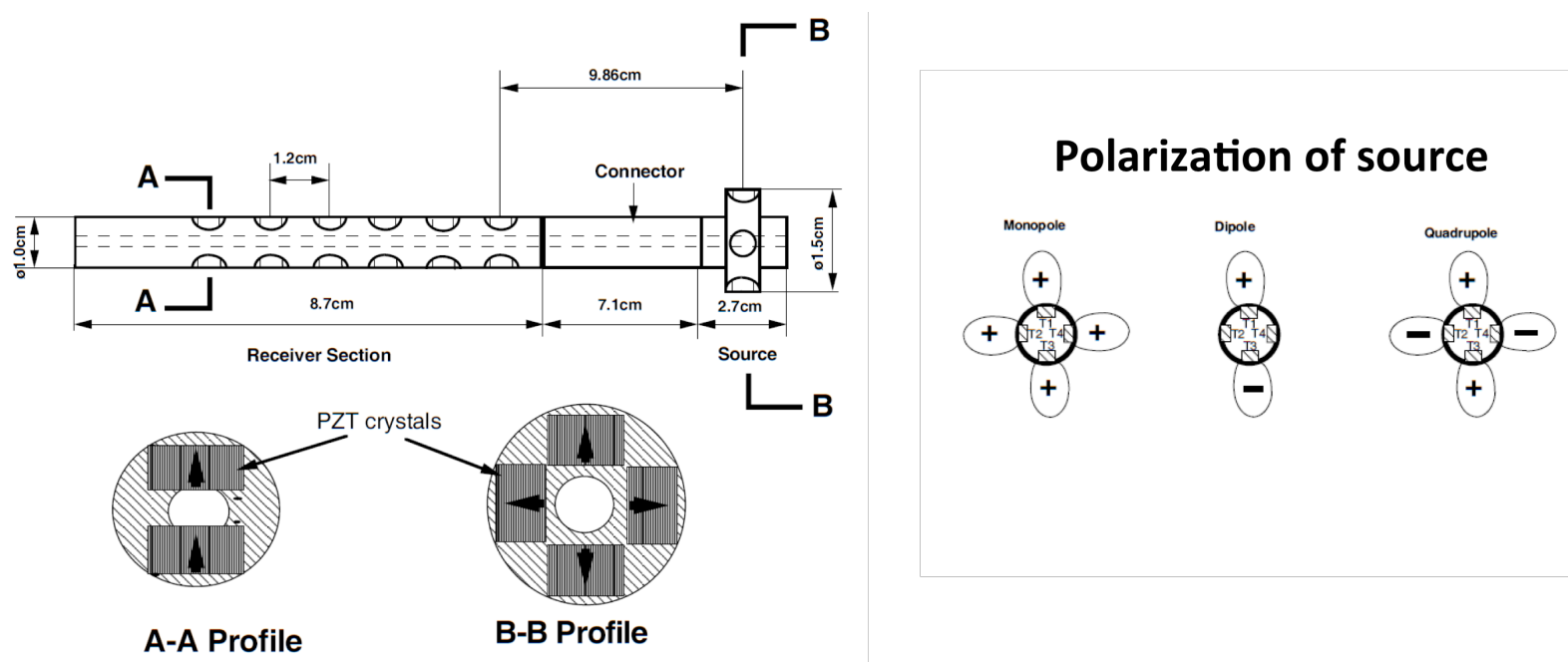

Figure 5: Schematic of a scaled multipole acoustic logging tool with a source and six receiver pairs. The single transducer is a PZT cylinder crystal that is $0.64 \mathrm{~cm}$ in diameter and $0.37 \mathrm{~cm}$ long. The radiation patterns are shown on the right side when the source works as monopole, dipole, and quadrupole, respectively. 


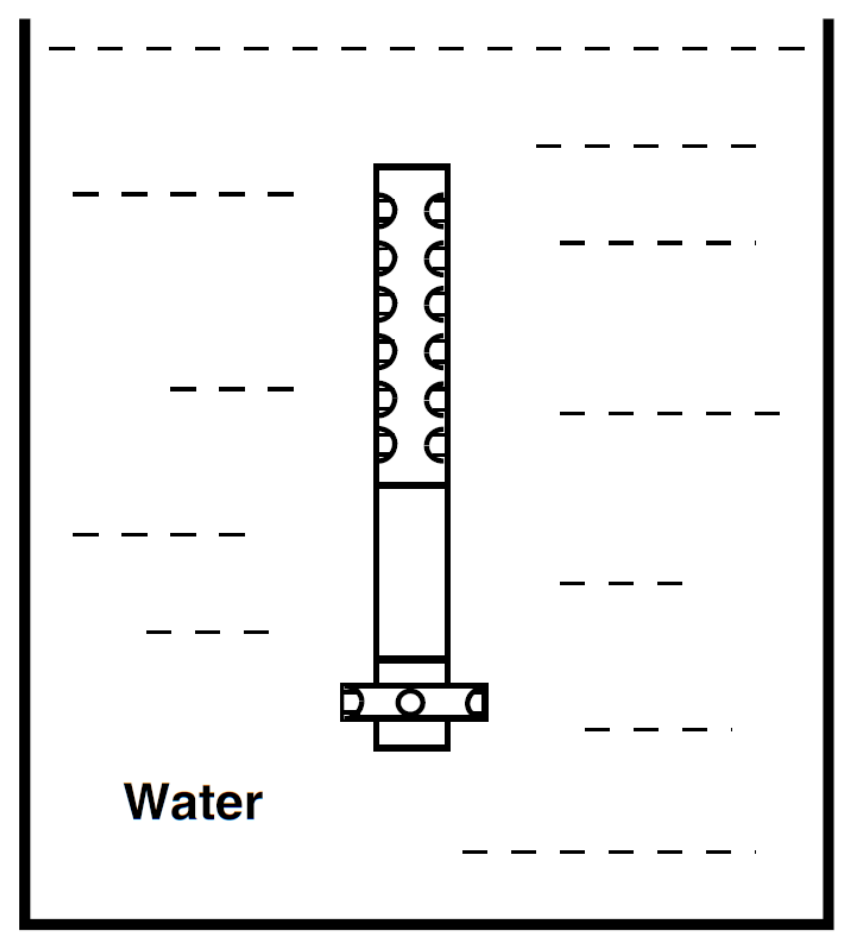

Figure 6: Schematic of the acoustic measurements in a water tank when the source and receiver section are tightly connected to simulate acoustic LWD tool. 

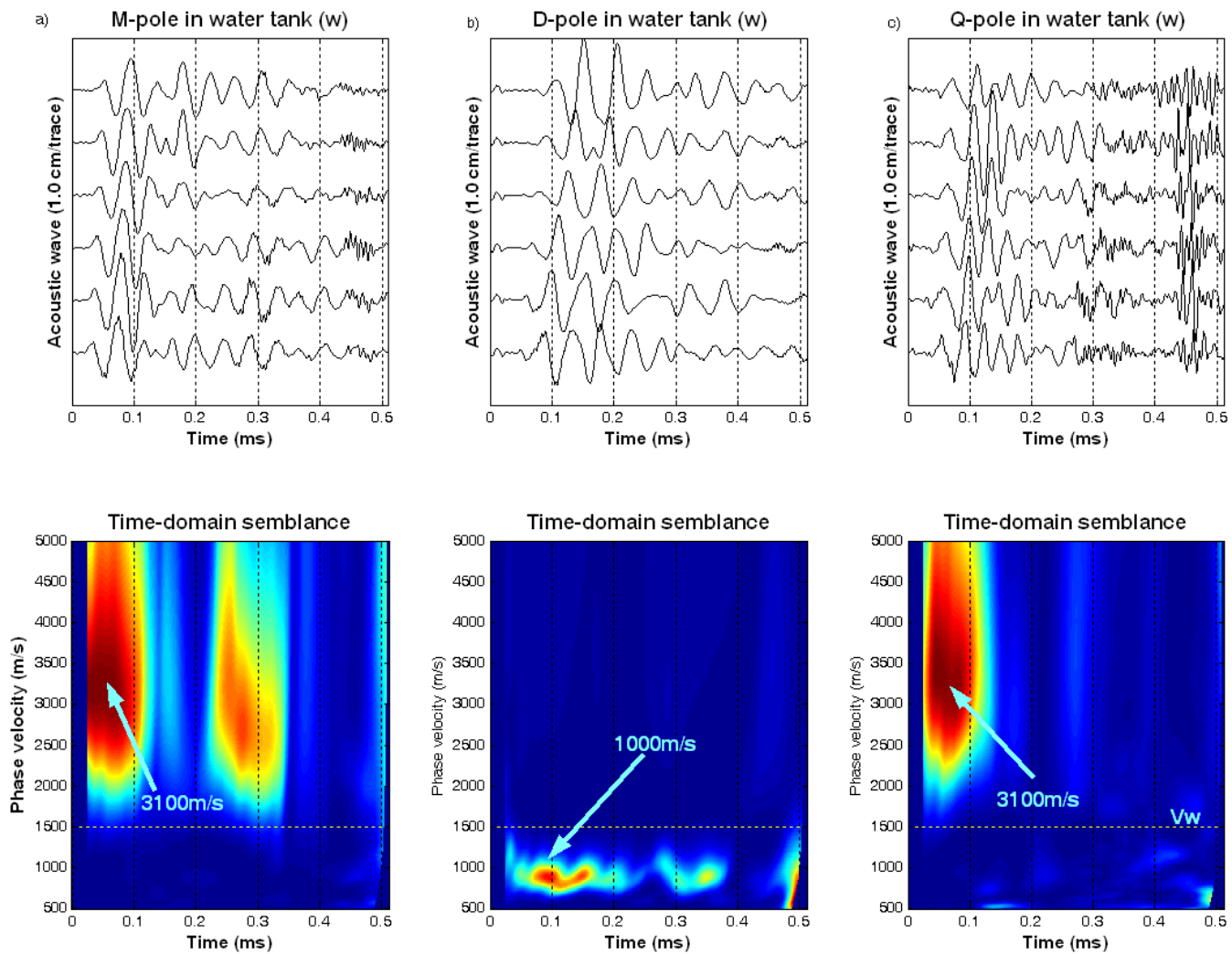

Figure 7: Acoustic waveforms and their semblance plots in the time domain when the multipole tool works in a water tank at (a) monopole, (b) dipole, and (c) quadrupole modes, respectively. The high-frequency signals received after $0.28 \mathrm{~ms}$ are the reflection from the boundary of the water tank. The source is excited by a single sine burst with $50 \mathrm{kHz}$ in center frequency. The dotted line $\left(V_{w}\right)$ indicates the water velocity. 


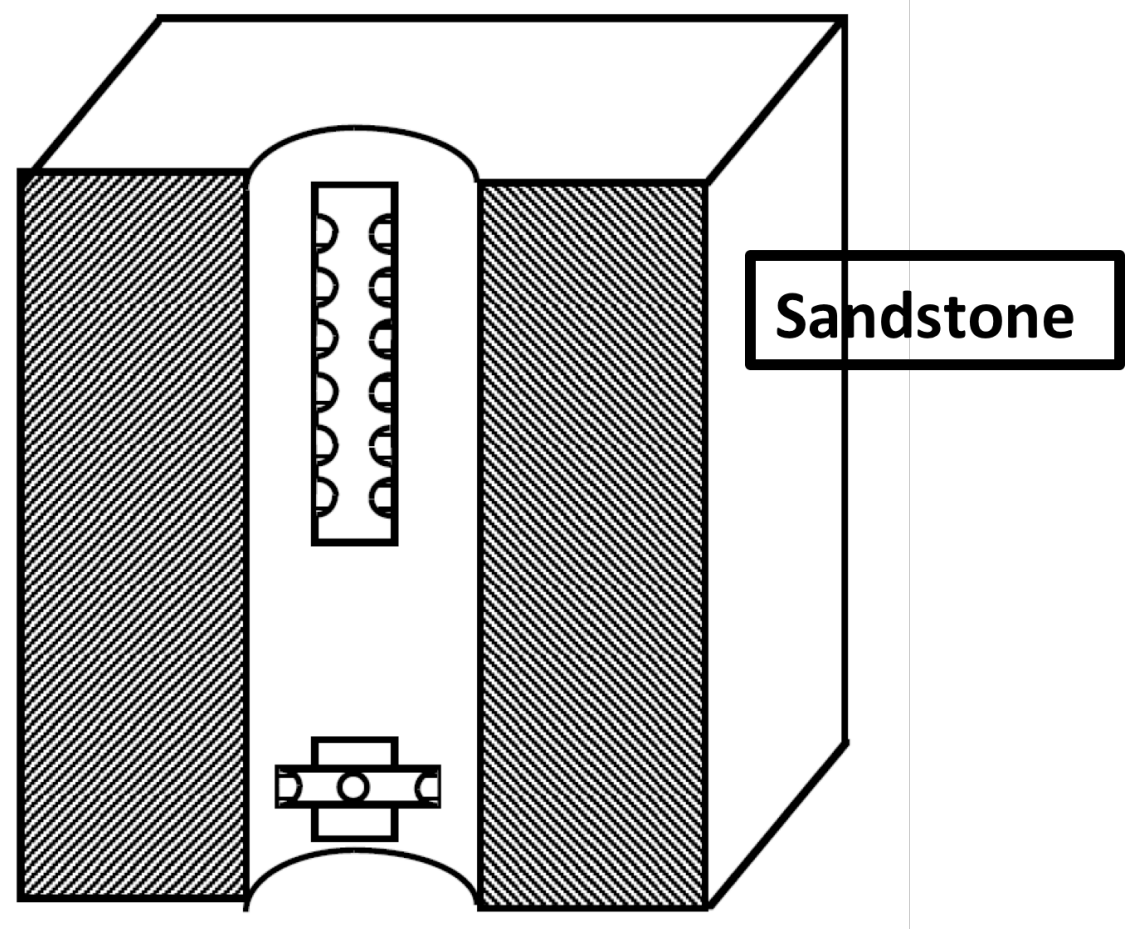

Figure 8: Schematic of the acoustic measurements in the sandstone borehole model with the tool without the connector between the source and receivers. 

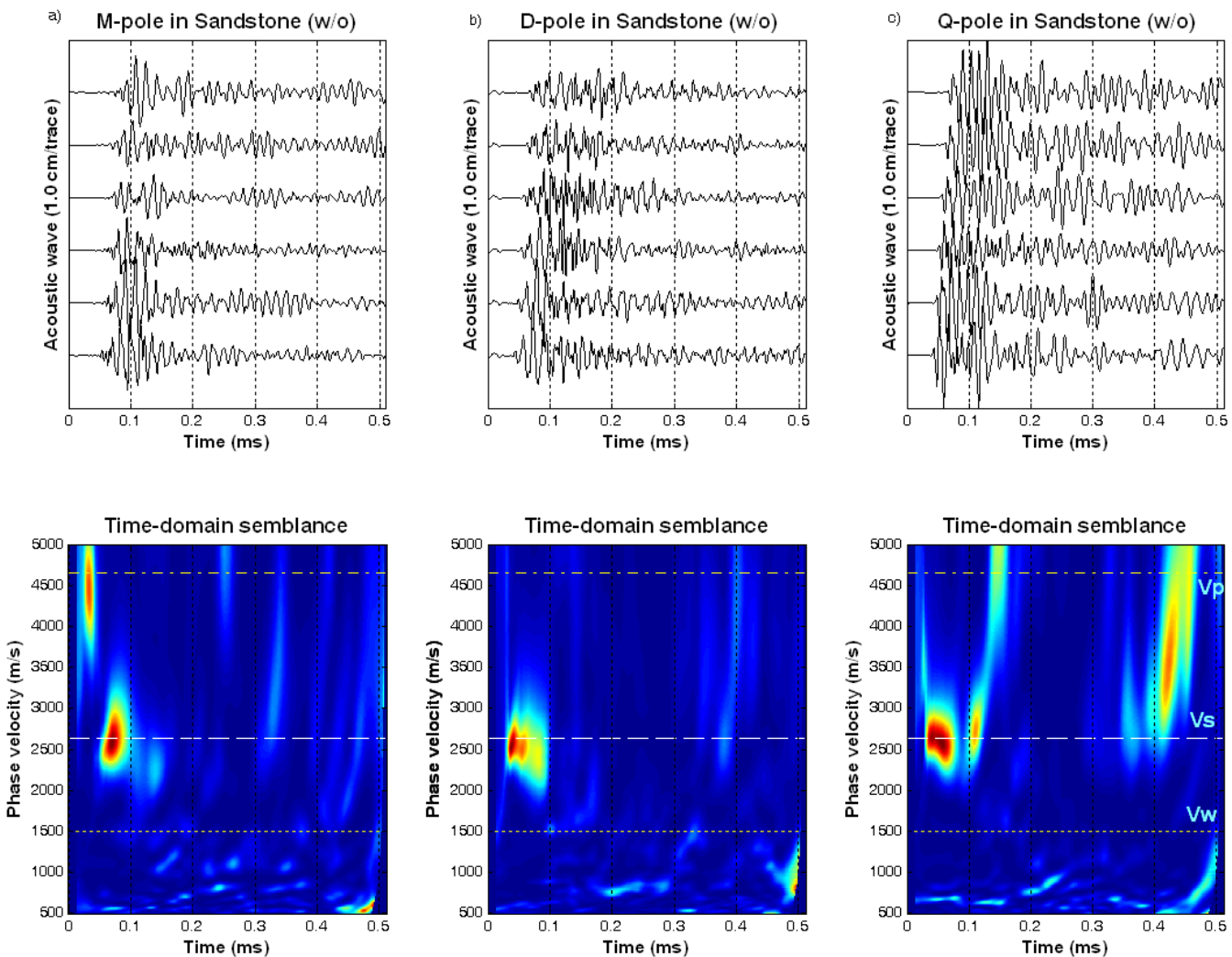

Figure 9: Acoustic waveforms and their semblance plots in the time domain when the multipole tool works in the sandstone borehole model at (a) monopole, (b) dipole, and (c) quadrupole modes without the connector, respectively. The dotted-dashed line $V_{p}$ and the dashed line $V_{s}$ indicate the $\mathrm{P}$ - and S-wave velocities in sandstone, respectively. The dotted line $V_{w}$ indicates the water velocity. 


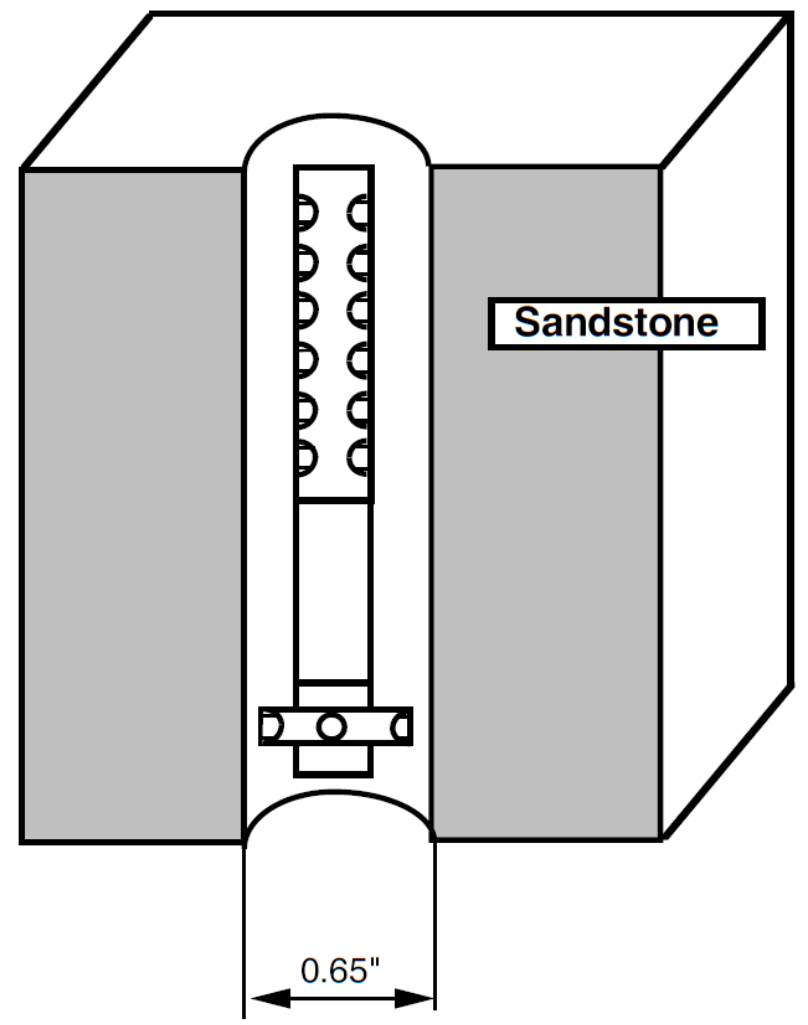

Figure 10: Schematic of the acoustic measurements in the sandstone borehole model when the tool it tightly connected with the connector between the source and receivers. 

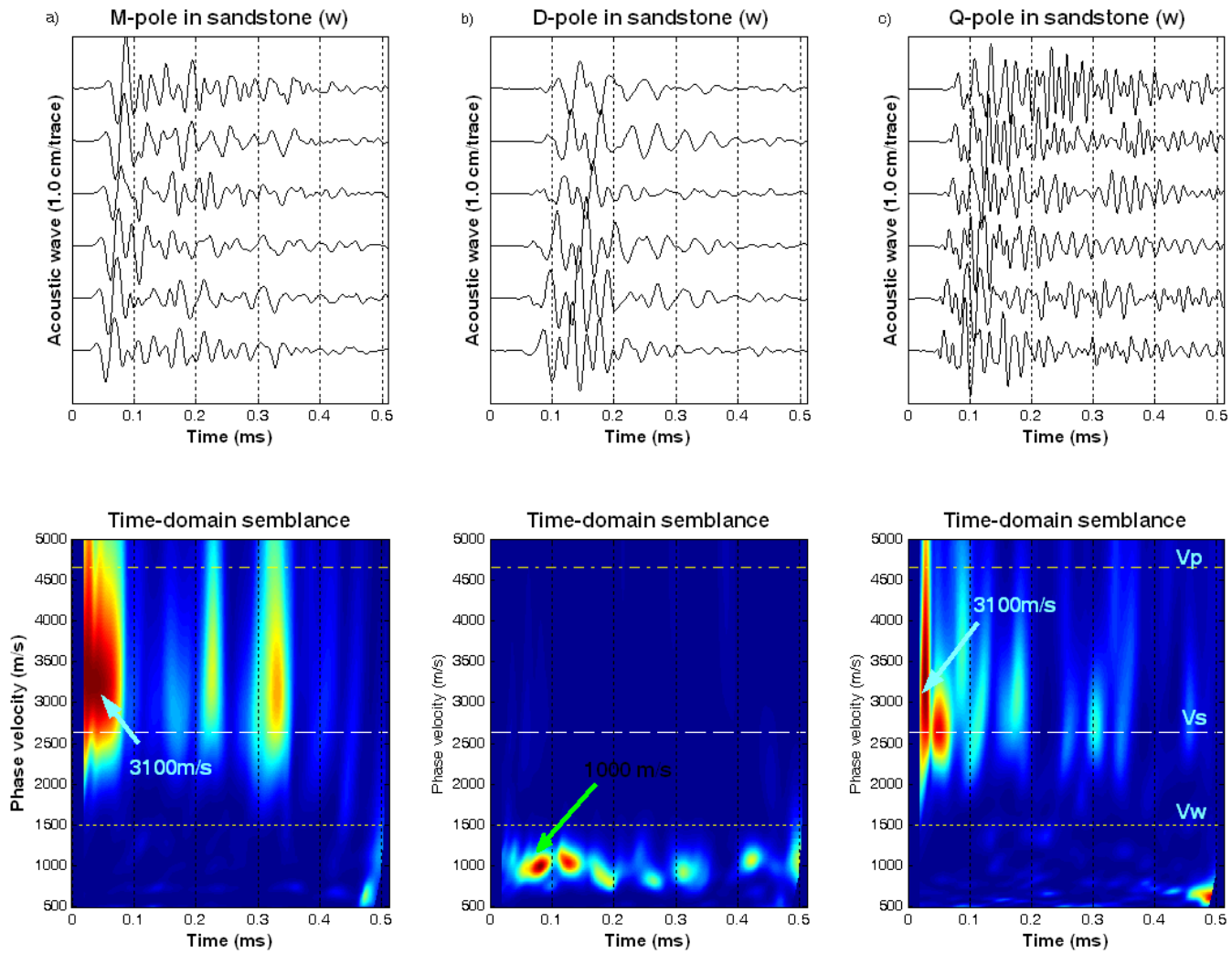

Figure 11: Acoustic waveforms and their semblance plots in the time domain when the multipole tool works in the sandstone borehole model (a) monopole, (b) dipole, and (c) quadrupole modes, with the connector between the source and receiver sections, respectively. The strong tool waves are recorded in the three modes. The horizontal lines of $V_{p}, V_{s}$, and $V_{w}$ indicate the P-wave, Swave and water velocities, respectively. 


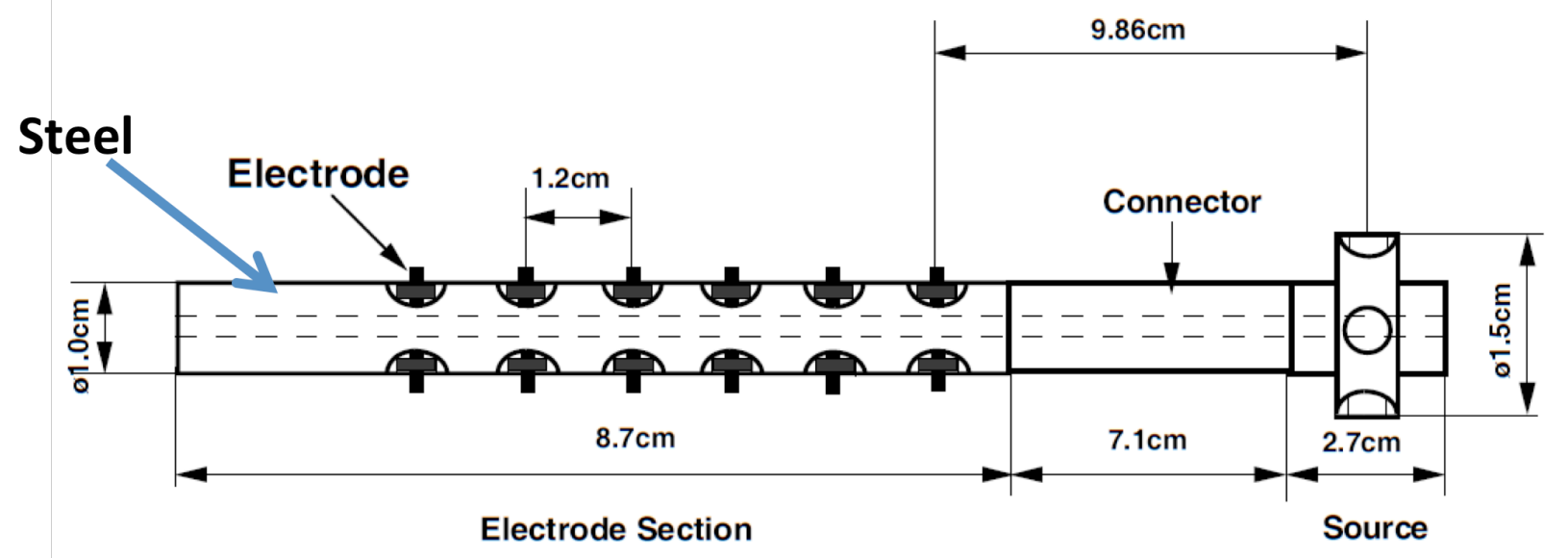

Figure12: Schematic of a scaled multipole seismoelectric logging tool with a source and six electrode pairs. The receiver crystals in Figure 5 are replaced with the electrodes. The source and the tool sizes are the same as the tool shown in Figure 5. 


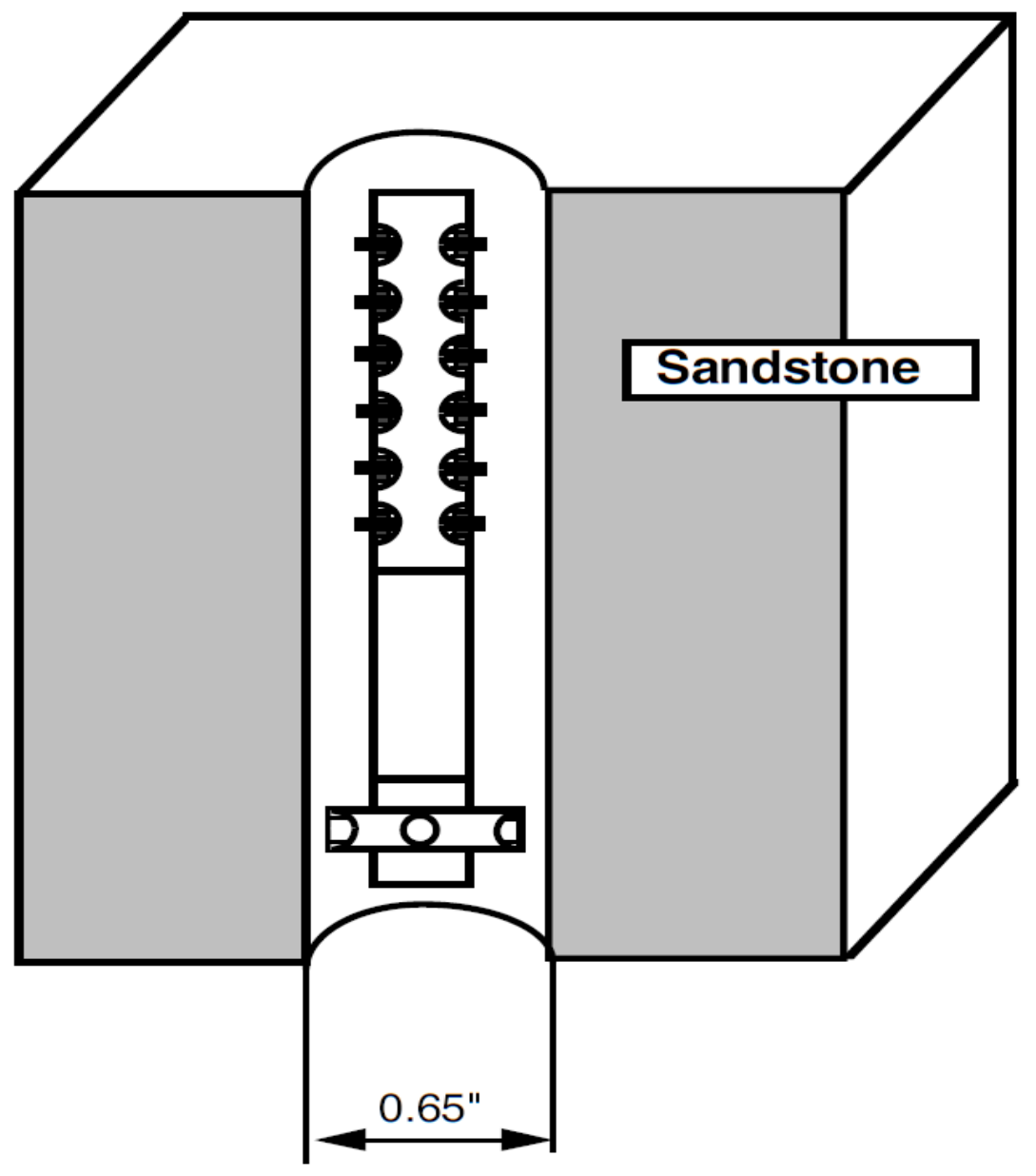

Figure 13: Schematic of the seismoelectric measurements in the sandstone borehole model when the tool is tightly connected with the connector between the source and receivers. 

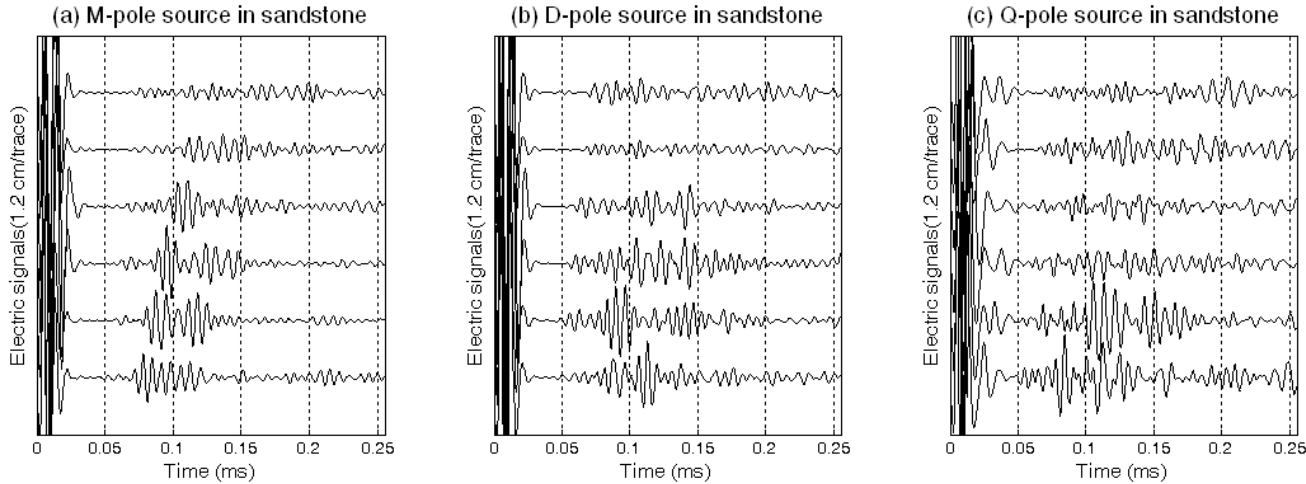

(a)Time domain semblance

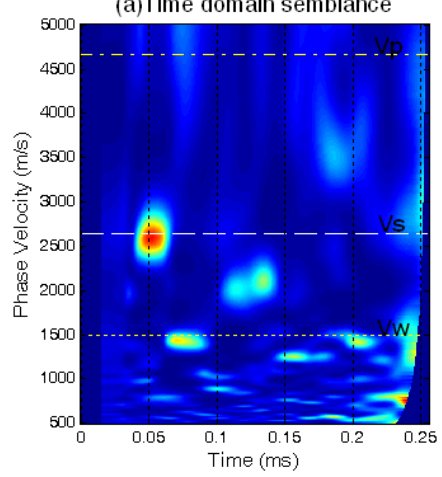

(b)Time domain semblance

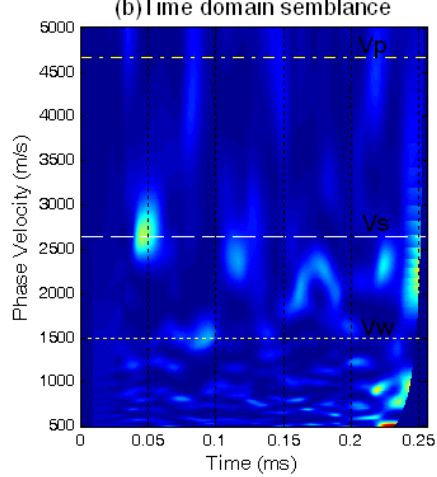

(c)Time domain semblance

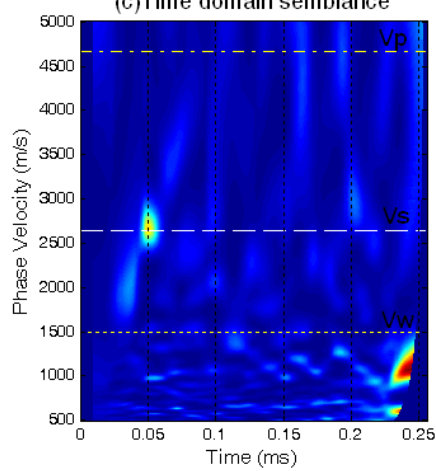

Figure 14: Seismoelectric signals and their semblance plots in the time domain when the multipole seismoelectric tool works in the sandstone borehole model as (a) monopole, (b) dipole, and (c) quadrupole modes, with the connector between the source and receiver sections, respectively. No electric signals with the apparent velocity of the tool wave are recorded. The horizontal lines of $V_{p}, V_{s}$, and $V_{w}$ indicate the $\mathrm{P}$-wave, S-wave and water velocities, respectively. 